Document downloaded from:

http://hdl.handle.net/10251/44404

This paper must be cited as:

Montagud, M.; Boronat Segui, F. (2014). Analysis, Deployment, and Evaluation of the Use of Network Simulation as a Learning Resource. Rita -IEEE. 9(3):82-90.

doi:10.1109/RITA.2014.2340011.

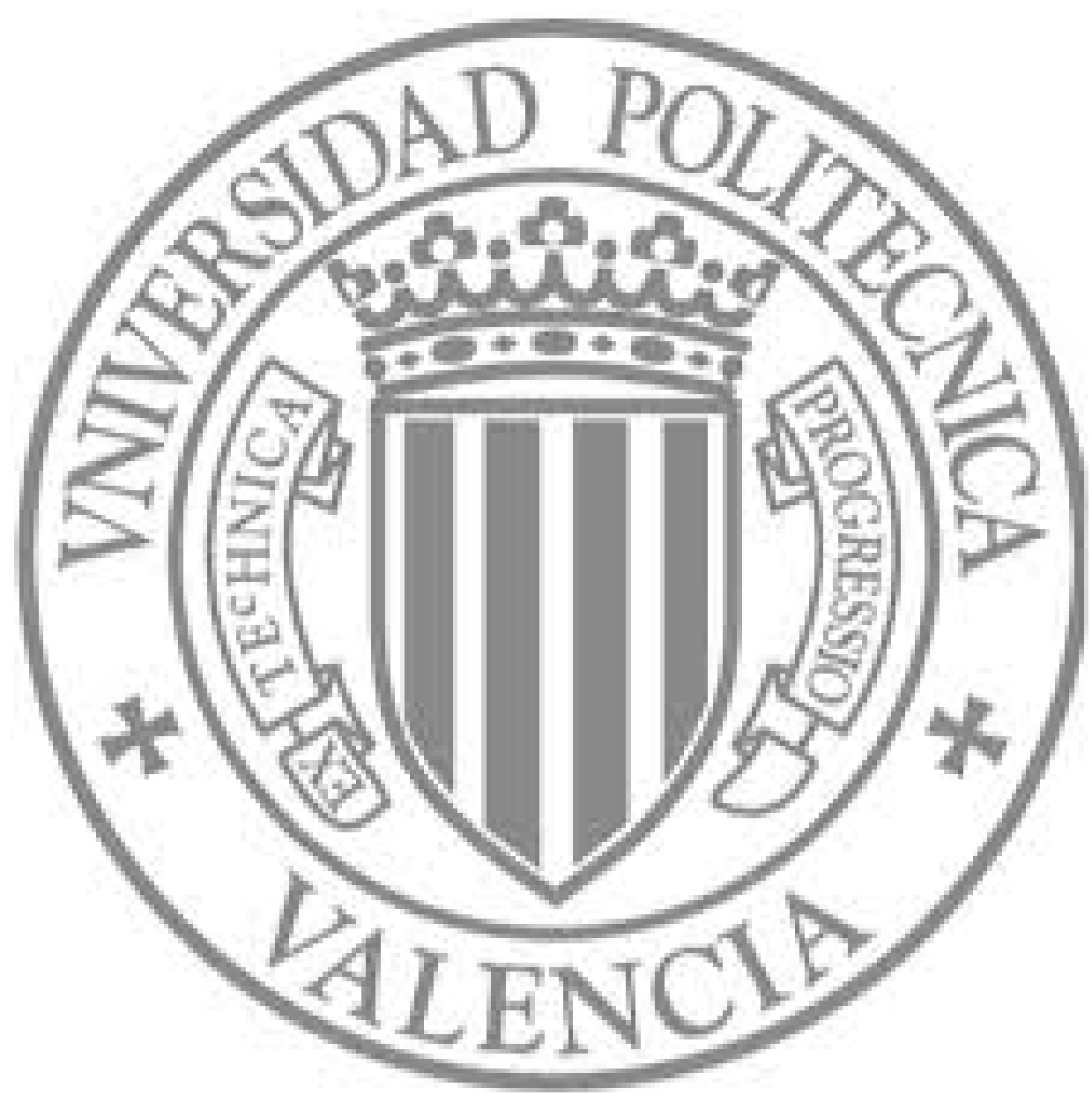

The final publication is available at

http://dx.doi.org/ 10.1109/RITA.2014.2340011

Copyright Institute of Electrical and Electronics Engineers (IEEE) 


\title{
Analysis, Deployment and Evaluation of the Use of Network Simulation as a Learning Resource
}

\author{
Mario Montagud, Student Member, IEEE, and Fernando Boronat, Senior Member, IEEE
}

\begin{abstract}
This paper analyzes the benefits of using network simulation as a learning resource in the educational area of Telematics Engineering. Accordingly, a (students' skills) practice program, which can be done by using either network simulation or real equipment, has been introduced in the core subject "Telematics Architectures and Networks (TAN)" of the second year of the Bachelor's Degree in "Telecommunications Systems, Sound and Image Engineering". The satisfactory evaluation of this innovative teaching methodology is proved by the rating of the surveys answered by the students after the course
\end{abstract}

Index Terms - Telematics Engineering, Computer Networks, Simulation, Learning/Training Resources, Practical Sessions

\section{INTRODUCTION}

$\mathrm{T}$ HE guidelines from the European Higher Education Area (EHEA) imply a new approach of the educational programs. Innovative educational methodologies must be introduced to strengthen the strategic thought of students and their ability to acquire knowledge, skills and attitudes that will help them to overcome future challenges during their professional careers.

The Bachelor's Degree in "Telecommunications Systems, Sound and Image Engineering", coursed at the Higher Polytechnic School of Gandia (HPSG), which is an external Campus of the Polytechnic University of Valencia (UPV), aims to train highly qualified Information Technology and Communications (ICT) professionals. Some of the key skills to be acquired by the students of this degree are strongly related to the area of Telematics Engineering (TE). In its educational program, we can find a block of subjects (see Table I) that aim, as a whole, to train the students in the areas of planning, designing, configuring, managing, maintaining and interconnecting different types of computer networks (e.g., LANs, WLANs, WANs, etc).

Traditionally, different learning methodologies (e.g., based on discovery, active learning, cooperative learning, etc.) have been employed at the HPSG in the TE area. These methodologies have been complemented to gradually provide students with proper training, while stimulating their theoretical and practical skills [1].

The authors are with UPV, 46730, Grao de Gandia (Spain) (e-mail mamontor@posgrado.upv.es, fboronat@dcom.upv.es).

DOI (Digital Object Identifier) Pendiente
TABLE I

SUBJECTS OF THE TELEMATICS ENGINEERING (TE) AREA

\begin{tabular}{|c|c|}
\hline Semester & Subject \\
\hline 1B & Fundamentals of Telematics \\
\hline 2B & Telematics Architectures and Networks (TAN) \\
\hline 2B & Telecommunication Networks and Systems 1 \\
\hline 3A & Telecommunication Networks and Systems 2 \\
\hline
\end{tabular}

Due to its highly technical nature, the acquisition of skills in the TE area cannot be uniquely based on memory-based learning. Accordingly, complementary learning processes (e.g., based on problems, use cases or practical activities) [2, 3] must be adopted to make it possible for students to better assimilate the theoretical concepts through their own experience. This way, the students will play a more active role in lectures. Likewise, they will assume a higher responsibility and develop critical minds, as well as their capacity for analysis, communication and planning.

In addition, the acquisition of skills in the TE area requires a continuous and gradual learning process. The risk of advancing towards the most complex concepts without having previously and solidly acquired the most basic ones must be minimized. Therefore, the students must be guided by an adequate monitoring process, providing them with simple and flexible learning tools to encourage their commitment and knowledge acquisition capabilities. Furthermore, these tools must provide a smooth transition between having no knowledge and learning the most advanced aspects of the TE area, allowing the students to get started, be gradually familiar with and, finally, become experts in this professional field after finishing their studies.

In this context, network simulation (software) frameworks become suitable pedagogical tools in the TE area to complement and enrich the students' learning and training processes. Network simulators allow to apply the theoretical concepts (explained in lectures) and to experiment with them, offering students an initial contact with the complex world of computer networks in a simple, flexible and relaxed manner.

This paper discusses the advantages of using network simulators as a learning and training resource in the TE area. Accordingly, a (students' skills) practice program is introduced to be integrated in the core subject "Telematics Architectures and Networks (TAN)" of the second year of the Bachelor's Degree in "Telecommunications Systems, Sound and Image Engineering” (see Table I). This subject is also part of the "Adaptation to the Degree" course, which is targeted at 
graduate students from the previous curricula. These practical sessions aim to exercise basic and advanced concepts of the network layer architecture of the OSI (Open System Interconnection) model. The rating of the surveys answered by the students after the course completion corroborates the benefits of adopting this innovative learning methodology in terms of flexibility, degree of autonomy, time saving, knowledge acquisition, and similarity with working with real equipment.

This paper is structured as follows. Next section discusses related work. In Section III, the advantages of using network simulation as a learning resource are described. Also, some guidelines for selecting an appropiate simulation tool are introduced. The key aspects and functionalities of the selected simulation framework for this course are outlined in Section IV. Section V presents the practice program and voluntary activities that have been prepared and deployed to complement and enrich the students' learning process for the concepts studied in this core subject. The evaluation criteria, the monitoring process of this practice program, and the students' impressions about the adoption of this learning methodology are described and analyzed in Section VI. Finally, Section VII is a conclusion and a discussion of future work.

\section{RELATED WORK}

In technical education, the assimilation of the theoretical concepts explained in lectures through an applied and practical way is essential. This aspect enriches, undoubtedly, the learning and skills acquisition processes. However, in the $\mathrm{TE}$ area, the deployment of laboratory practical sessions that allow students to experiment with real network scenarios is often not feasible (or too costly), mainly due to their complexity, their spatial diversity and/or the limited availability of material resources. The study in [4] aims to show the difficulties of deploying laboratory practice in this field. On the one hand, it is not easy to monitor the particular progress and the completed tasks for each student in groups involving a large number of people. On the other hand, the realization of practical tasks involves a higher dedication and lower flexibility by the instructor for checking the achievement of the intended objectives. It is also important to mention the study in [5], in which a high percentage of students considered that the duration of the laboratory practicals by using real equipment was too short, because many times they were not able to finish them, especially if something came up during their evolution, such as using inadequate or broken cables/connectors, incorrect or uncomplete configurations, uncoordinated tasks, etc.

In this context, network simulators offer the possibility of analyzing the practical aspects of communication services and protocols without needing to deploy real network scenarios and infrastructure. As it is described in the next section, the use of network simulators is advantageous in both the academia and professional fields. For that reason, different teaching innovation projects have adopted network simulation as a learning resource. On the one hand, a web-based network simulation tool was presented in [3] to allow students to get started in the computer networks field in a simpler and more flexible manner. This work states that most of the simulators are oriented to the professional and scientific fields and their use as an educational resource can overwhelm novice students. On the other hand, the use of network simulation to facilitate and to enhance the students' learning process in an active and stimulating way is proposed in [6]. The benefits provided by this methodology with regard to knowledge acquisition and to the capacity for configuring networked scenarios are statistically assessed through surveys answered by students, both before and after the completion of the practical sessions.

The novelty of this paper with respect to the above contributions is that the prepared practice program and volunteer activities can be done either through simulation or with real networking equipment. Accordingly, the advantages of both methodologies can be complemented. Likewise, the students can check the feasability and functionality of the analyzed scenarios through the simulator but they also deploy these scenarios by using real and comercial equipment in the laboratory. Finally, the conducted tests are not only intended to determine the students' learning level in each practical but also to gather their opinion regarding other important aspects (see Table IV), such as the degree of flexibility and autonomy provided by the use of network simulation, the difficulty level to become familiar with the selected network simulator, the similarity with the work by using real equipment, as well as their opinion about the content, duration and the number of scheduled practicals.

\section{Advantages of Using Network Simulators}

Nowadays, network simulation tools are widely employed in the academic and scientific fields, but also in the professional field. In academia, the use of network simulators can provide the following advantages:

- They allow to complement blackboard, projector and/or paper activities with more practical ones that enable to apply the studied theoretical concepts without needing to deploy physical scenarios.

- They provide an initial contact with communication networks in a flexible, modular, and global way. This is possible because all the design and configuration issues of the computer networks components can be controlled from the simulation platform.

- They are very useful educational tools for learning to distinguish different networking components and their purpose, the most common commercial products, how they must be configured to achieve specific objectives, the basics about functional network architectures, as well as about existing standards and protocols related to the design, deployment and interconnection of computer networks.

- They are an alternative training resource in those cases 
where practicing with real equipment may be unfeasible or too costly (in terms of time and/or material availability). By using them, the installation, configuration and analysis processes of large-scale complex computer network are considerably simplified.

- They encourage autonomous work and training, and also provide higher flexibility. For example, if students cannot attend a practical (due to a justified cause) or are unable to finish their work during the scheduled time slot for it, they can save their configuration files and continue their work later, resuming the last task they completed. In addition, instructors can retrospectively evaluate the configuration files of each student in a more flexible and efficient way, which allows them to track the students' work and to check the assimilated concepts, as well as to identify possible conceptual shortcomings in their learning proceses.

- Their use allows to eliminate availability and accessibility barriers to the laboratory resources. Students can practice anywhere and anytime. In this way, they can repeat the practice sessions as many times as they wish, as well as to deeply analyze the studied concepts by adding advanced functionalities. Although laboratory practice encourages teamwork (since students must share the available resources and schedule the required tasks to deploy advanced network topologies), it limits the flexibility and autonomy of students. In some cases, individual work is essential for an adequate progress.

On the other hand, network simulators also play a key role in the professional field:

- They can be used as a strategic tool in the design process. Before the deployment of a real system, the suitability of various alternatives, regarding the architecture, technologies, protocols, applications or equipments to choose, can be analyzed without the need for any physical infrastrucuture. Therefore, they are fundamental support tools for decision making, since they allow to predict and/or assess the impact of any decision on the behavior of the system before being deployed.

- They are very useful tools to conduct measurements and requirement studies for the network infrastructure. By using them, the feasibility of experimental deployments can be assessed and pre-configuration tasks can be proved.

- They also allow analyzing the performance and behavior of a real system by monitoring some parameters of interest, without having neither to temporarily interrupt their operation nor compromise the resources of the network infrastructure. Performance tests, QoS measurements, etc. can be assessed more easily and with higher flexibility, while controlling the evolution of the system under anomalous situations that can be forced without involving cost or prejudice (e.g., links or equipment failures, network congestion, etc.).

\section{A. Selection of the Simulation Platform to Use}

Nowadays, we can find a huge variety of network simulation tools, such as OPNET [7], Omnet [8], NS-2 [9], NCTU [10], Packet Tracer [11], GNS3 [12], etc. Some previous works (e.g., [13]-[15]) have addressed comparative studies between existing network simulators taking into account different perspectives: suitability for academia, statistics of use in research studies, technologies support, reliability and accuracy of the implemented modules, opensource characteristics, etc.

In [14], a formal procedure for evaluating and selecting the most appropriate simulation tool in a reasoned and impartial way is proposed. This method is based on two key elements: i) the ISO/IEC 9126-1 norm, which specifies a quality and evaluation model for software tools; and ii) a cost function which analyzes the weight of different parameters defined in this standard, such as functionality, reliability, usability, efficiency, tasks maintenance and portability.

The goal of this paper is not to provide a comparative study between existing network simulators, but to present and briefly discuss some basic criteria that must be taken into account for choosing a specific simulator for the academic context.

First, the ease of use is essential. It would not be optimal to use an advanced, but complex, simulation platform, such as NS-2 [9], for specific academic tasks (e.g., for practical sessions of limited duration). This kind of simulators is better suited for conducting larger and long term projects, such as Degree's Final Projects (DFPs), Master Theses or research studies. A simulation platform ensuring a quick learning curve must be chosen, because if the simulation tool is simple and intuitive, the concepts will be assimilated more fluidly.

Second, the configuration tasks in the network simulator must provide the students with a degree of realism similar to the associated tasks when real equipment is employed.

Third, the software must implement accurate simulation modules and libraries, so that the simulation tests can accurately reflect the behavior of real communication systems.

Lastly, the simulation tool to choose has to be flexible enough to be adapted to the learning context in which it is intended to be used, supporting from the most basic concepts to the most advanced ones of the TE area, while providing a smooth and relaxed transition towards the work with real networking components.

In the next section, the basic characteristics of two of the simulation platforms that better meet the above requirements and, therefore, are very adequate for practicing with the concepts studied in the TAN subject, are presented.

\section{PACKet TRACER Characteristics}

Packet Tracer $(P T)$ is an interactive simulation tool used as a learning resource in the Cisco Networking Academy Program [16] for the preparation and training of students in the diverse courses and certification levels (e.g., Cisco CCNA, CCNP, CCIE ...).

This network simulator allows the users to configure diverse network topologies through a very intuitive graphical interface. PT supports a lot of networking components and devices, either generic or specific Cisco models, such as hubs, 
switches, routers, Network Interface Cards (NIC), access points, firewalls, printers, IP Phones, PCs, servers (e.g., HTTP or FTP, with the aim of simulating data transactions between communication devices), as well as different types of (wired and wireless) physical links. Table II summarizes the main functionalities, protocols and technologies supported by PT, for each layer of the TCP/IP protocol stack.

By using PT, users can have access to emulated usual tools and functionalities in each one of the components of the network topology under configuration, such as a web navigator, a command console, remote access configuration or graphical NIC configuration.

The devices can be configured through either an emulated graphical interface or an emulated command console through a Command Line Interface (CLI), supporting most of the Cisco IOS (Internetwork Operating System) commands. Therefore, the students can practice with the network topologies being configured as if they were working with real equipment, with a high degree of realism and accuracy.

Moreover, as the students can experiment with the configuration of commercial products (in this case Cisco's) in a similar way to how they would proceed by using real equipment, this enables the students to value the program of the TAN subject and its application to the professional environment.

Once the physical and logical configurations of each device have been completed, connectivity tests can be performed (e.g., by either using configuration checking commands, such as show or debug, or by issuing ping or traceroute commands) with the goal of testing the correct configuration and operation of the network topology under analysis.

PT supports different operation modes: topology, real-time and simulation. In particular, the simulation mode is very adequate for teaching purposes because it allows monitoring, with a high degree of control, the traffic flow and the sequence of operations originated in the network, as a consequence of the triggering of specific events, such as issuing a ping command or requesting a web page. For instance, the processes of origination, destruction and reception of the packets, as well as the paths they follow to reach the target destination/s, both step-by-step and in animation mode, can be inspected. Moreover, users are able to filter the monitoring of specific protocols and check the sequence of processes that are originated for a specific packet in their way for each component in each layer of the TCP/IP stack, as well as to inspect the content of the packet's header, how decisions are made, etc. In case of failure, the last successful processes can be looked for, with the aim of identifying configuration incompatibilities or errors.

PT also includes some advanced functionalities, such as the multi-user extension, which allows developing simultaneous collaborative practice sessions between networked users, so that the required tasks to configure complex network topologies can be divided among them, sharing their schemas and configuration files. Therefore, PT is also a useful tool for enforcing teamwork, as well as for stimulating planning, cooperation, leadership, and communication skills through the exchange of impressions and discussion of alternatives, prior to decision making.

TABLE II

SUPPORTED FUNCTIONALITIES BY PACKET TRACER (PT)

\begin{tabular}{|c|c|}
\hline TCP/IP Layer & Protocols/Technologies \\
\hline \multirow{3}{*}{ Application } & $\begin{array}{c}\text { HTTP, HTTPs, Telnet, SSH, TFTP, SMTP, POP3, } \\
\text { DHCP, NTP, DNS, SNMP, AAA, Radius, } \\
\text { TACACS, Syslog, ... }\end{array}$ \\
\hline Transport & TCP/UDP, ... \\
\hline \multirow{2}{*}{ Network } & $\begin{array}{c}\text { Static and dynamic routing }(\mathrm{RIPv} 1 / \mathrm{v} 2 / \mathrm{ng}, \text { EIGRP, } \\
\text { Multi Area OSPF, routes distribution...), Multi- } \\
\text { layer Switching, ... }\end{array}$ \\
\cline { 2 - 2 } & $\begin{array}{c}\text { Addressing: IPv4, IPv6, ICMPv4 e ICMPv6, ARP, } \\
\text { IPSec, NAT, ... }\end{array}$ \\
\hline \multirow{2}{*}{ Sub-network } & Ethernet 802.3, VLANs, 802.11, 802.1q, HDLC, \\
& $\begin{array}{c}\text { PPP, Frame Relay, ISDN, STP, RSTP, VTP, DTP, } \\
\text { CDP, PAgP, LACP, EAP, WEP, WPA, ... }\end{array}$ \\
\hline
\end{tabular}

Therefore, by using PT, students can simulate from very simple networks, with minimal configuration parameters, to very complex network topologies with many potential configurations. PT provides high flexibility in the learning process, complementing the classroom and laboratory tasks, allowing the students to experiment and discover, in an active, creative and autonomous manner, the functioning of computer networks. Moreover, PT is a very useful platform for teachers or instructors to demonstrate and illustrate the operation of diverse components, protocols and technologies of specific communication networks.

However, the use of PT has some drawbacks. Its license use is restricted to registered users in the Cisco Networking Academy. Furthermore, PT does not support some advanced functionalities and commands of the Cisco devices and last IOS releases, respectively. For that reason, a possible alternative to PT is GNS3 [12], which is a more advanced, open-source, and multi-platform graphical simulator/emulator that allows experimenting with complex networks.

Using GNS3, real networking components of referent manufacturers (e.g., Cisco or Juniper) can be virtualized by loading and executing the images of their IOS. It is important to note that GNS3 does not include these images (the simulation platform is free but the images of the networking components are not). A very important characteristic is that GNS3 allows the communication between physical and virtual networks, as well as capturing the packets traversing the virtual links. Therefore, GNS3 is a more potent, but also more complex to use, platform than PT, which can also be used as a learning and training resource for advanced students and professionals of the TE area.

\section{Practice Program for the TAN SubJect}

The TAN subject has a workload of 6 European Credit Transfer and Accumulation System (ECTS), divided into 4 credits of theoretical contents and practical exercises and 2 credits of laboratory practice. On the one hand, the theoretical aspects are explained through lectures and their analytical application is intended to be inculcated through problems and 
use cases formulation, as well as through classroom activities. On the other hand, their practical application is trained in laboratory sessions, such that students can learn through their own experience. The agenda of the TAN subject is intended to give a general overview of the layered network architecture, mainly focusing on the internal organization of the network layer, its basic functions and the offered services. The aim of this subject is to train the students in general network interconnection issues, particularly focusing on the solution provided by TCP/IP protocol stack. Moreover, some advanced IP services are studied, such as policies and solutions for optimizing the IP address space (e.g., subnetting, Network/Port Address Translation or NAT/PAT), dynamic address assignemt mechanisms (Dynamic Host Configuration Protocol or DHCP), address resolution mechanisms (Address Resolution Protocol or ARP), as well as different routing policies, such as those based on distance vector or link state techniques (presenting some examples of representative protocols such as Routing Information Protocol or RIP, and Open Shortest Path First or OSPF, respectively). Finally, general aspects and the basic structure of packet-switched Wide Area Networks (WANs), as well as real use cases of point-to-point connections, either through direct (or leased) lines (by using Point-to-Point Protocol or PPP) or virtual circuits (by using Frame-Relay Permanent Virtual Circuits or $P V C s)$, are studied.

\section{A. Laboratory/Simulation Practice}

A total number of ten 2-hour sessions of laboratory practice have been scheduled (there are 8 different practicals, but 2 of them are scheduled to be completed in 2 sessions). Each practical consists of a brief introductory explanation, followed by configuration exercises and brief questions in an uninterrupted way. Six of these practicals can be done either by using real equipment or by using each one of the above presented simulation platforms (PT or GNS3).

Each one of the practicals is done after the explanation of the associated theoretical concepts in lectures. This facilitates their comprehension, as well as its previous study with the aim of maximizing a better use of the practices. Moreover, the students can access the practicals' documentation early enough before their realization, allowing them to read and prepare them in advance. The documentation includes strategic questions before and after analyzing the functioning/behavior of the different mechanisms and techniques under study. This undoubtedly will help the students to stimulate their capacity for prediction, to identify probable conceptual failures, as well as to assimilate in a solid way their knowledge acquisition processes.

The documentation of the first practicals is very complete and self-contained. As the course advances, the tasks to be completed in the practice sessions are exposed without detailing the exact steps to follow or the specific models of the networking components to use, letting some flexibility to the students to deploy the targeted assemblies. This way, the creativity and decision-making skills of the students are stimulated, since they should be able to find out which components meet the targeted requirements, being possible to achieve the objective by selecting different components. This policy requires the teacher to be more involved because he/she must control that students choose adequate components and guide/correct them in case of doubt/mistake. At the end of the practicals, the proposed alternatives, as well as other solutions proposed by the teacher, with their respective advantages and disadvantages, are discussed and compared. This way, if the practicals are done through simulation, their realization is not limited by the available real equipment in the laboratory.

In our laboratory, the necessary networking components to deploy each one of the proposed practicals are available. However, when the number of students is high the available resources must be shared. This fact can involve that the students consider that the laboratory facilities and resources should be improved in specific cases, as reflected in [5]. By using simulation, this limitation is overcome, as discussed in Section III.

The contents of the practice sessions have been prepared such that the concepts are introduced in a gradual way, to make the students' adaptation and learning processes easier, allowing them to solidly acquire the studied concepts, as well as to get familiar with their configuration processes in realworld networking components. Moreover, as the complexity of the practicals increases with the evolution of the course, the concepts learned in previous sessions will be pre-requirements in the successive ones. This stimulates an intregrated knowledge of the studied concepts in the TAN subject. Likewise, the necessary network topologies for each practical become gradually more complex as the course advances. In the simpler sessions, no configuration files are provided, while in the more complex ones, involving large-scale topologies and advanced services, pre-configured files are provided. On the one hand, this strengthens the skills acquisition regarding the configuration of networked scenarios but, on the other hand, this also helps students to reduce the time needed to complete the most laborious practice.

Even though the tasks of the practicals can be completed in the time slot scheduled for each one of them, if the students are unable to finish them or intend to study the concepts in more depth, they can save their configuration files and continue their work later, resuming the last task they completed. This is an important advantage of the use of network simulation. However, the instructor must also value the ability ot the students to complete the required tasks during the scheduled time slots for each practice session.

The prepared practicals, in conjunction with the intended learning objectives, are listed below:

Practical 1) Basic DHCP Configuration: Basic configuration of PCs (connectors, interfaces, NICs, IP configuration); basic configuration of routers (connectors, interfaces, NICs, IP configuration, clock rates, static/default/dynamic routing...); configuration of DHCP services (with addresses allocation), both in local and remote servers; DHCP process monitoring and debugging; and 
troubleshooting.

Practical 2) Basic Configuration of NAT and PAT: Basic configuration of PCs and routers; configuration of static and dynamic NAT, both with and without port overloading; configuration of Access Control Lists (ACLs); connectivity verification; NAT process monitoring; NAT events debugging; and troubleshooting.

Practical 3) Basic Routing Configuration using RIP : Basic configuration of PCs and routers; configuration of RIP (versions 1 and 2), and static routes; routing tables monitoring; RIP process monitoring and RIP events debugging; connectivity verification; and troubleshooting.

Practical 4) Basic Configuration of IPv6 (Internet Protocol, version 6): Basic IPv6 configuration of routers; basic configuration of static and dynamic routing using RIPng (RIP next generation); connectivity verification between IPv6 devices; routing tables monitoring; RIPng process monitoring; RIPng events debugging; and troubleshooting.

Practical 5) Basic Configuration of OSPF: Configuration of of basic and multi-area OSPF (version 2); adjacency relationships and routing information exchange monitoring; routing tables monitoring; connectivity verification; OSPF process monitoring; OSPF events debugging; and troubleshooting.

Practical 6) Configuration of WAN point to point Connection. Point-to-Point Links and Frame Relay PVCs: Change of the default High-Level Data Link Control (HDLC) encapsulation in the serial interfaces of Cisco routers by PPP encapsulation; configuration of authentication protocols (Password Authentication Protocol or PAP and Challenge Handshake Authentication Protocol or CHAP) for PPP encapsulation; connectivity verification; configuration of Frame Relay PVCs; monitoring and troubleshooting.

For each practical, the students must deliver an individual report reflecting the completed tasks, the answers to the formulated questions and the obtained results, in addition to the corresponding configuration files. In such reports, it is intended that the students synthesize the learned concepts and corroborate the successful exploitation of the practice sessions.

\section{B. Complementary/Voluntary Activities}

On the one hand, as addtional work, the reports of the practicals can be complemented with elaborated documentation of the implemented services, a detailed guide of the configuration processes and the selected equipment, budgets, comparative studies, etc. Moreover, a real reproduction of the practicals can be performed, by using real deployment in the laboratory, so that the students can corroborate the utility of the simulators to help them to learn to design and deploy computer networks. On the other hand, the students can also do voluntary tasks based on simulation, with the aim of (more deeply) studying some interesting concepts that are either not included in the TAN agenda or only superficially explained by the professor. A list of possible works is provided to the students, which is extensible by their own proposals. These works will be briefly presented to the rest of the students in the classroom with the goal of sharing the learned experiences and derived conclusions.

\section{Results}

Each cycle completion must involve an evaluation of impressions and an assessment of results. Both factors are important since the users' satisfaction and the benefits provided by the deployment of the proposed methodology will determine its success.

That is why, after the course completion, an anonymous survey was delivered to the students in order to collect their opinion about some key factors, such as level of complexity, spent time, flexibility, degree of autonomy, realism of the simulation tools and knowledge acquisition. The list of aspects to assess and their scoring levels (on a 0-4 scale) to measure the degree of satisfaction/approval of each individual student for each factor are in Tables III and IV, respectively.

The results, for a total number of 59 students during the 2011/2012 course, are shown in the pie charts of Fig. 1.

The opinions of the students regarding the first aspect show their complete agreement with regard to the number of planned practice sessions. Regarding the second aspect, most of the students were satisfied with the duration of the practicals, although a significant percentage (20\%) partially disagreed with this. However, without the flexibility and the higher degree of simplicity provided by simulators, we think this percentage would have been higher, as occurred in the study in [5].

TABLE III EVALUATION METRICS

\begin{tabular}{|c|c|}
\hline Opinion & Punctuation (0-4) \\
\hline $\begin{array}{c}\text { I do not have sufficient evidence to form an } \\
\text { opinion }\end{array}$ & 0 \\
\hline Totally disagree & 1 \\
\hline Partially disagree & 2 \\
\hline Partially agree & 3 \\
\hline Totally agree & 4 \\
\hline
\end{tabular}

TABLE IV

QUESTIONNARIES ABOUT THE PRACTICALS

\begin{tabular}{|l|l|}
\hline \multicolumn{1}{|c|}{ Indicative Aspects to be Evaluated } & Valuation \\
\hline 1. The number of practicals is adequate. & \\
\hline 2. The duration of the practials is adequate. & \\
\hline $\begin{array}{l}\text { 3. The content of the practicals corresponds to the } \\
\text { theoretical concepts studied in lectures. }\end{array}$ & \\
\hline $\begin{array}{l}\text { 4. The effort to complete the practicals has been } \\
\text { compensated by the knowledge and skills acquisition } \\
\text { related to the concepts studied in the course. }\end{array}$ & \\
\hline 5. The simulation platform is simple and intuitive. & \\
\hline $\begin{array}{l}\text { 6. The use of simulators has allowed me to consolidate } \\
\text { and deepen the theoretical concepts. }\end{array}$ & \\
\hline $\begin{array}{l}\text { 7. The practicals through simulation have allowed me } \\
\text { higher flexibility and autonomy. }\end{array}$ & \\
\hline $\begin{array}{l}\text { 8. The practicals through simulation accurately reflect } \\
\text { the work with real equipment in the laboratory. }\end{array}$ & \\
\hline $\begin{array}{l}\text { 9. I am satisfied with the experience and knowledge } \\
\text { acquired through the realization of the practicals. }\end{array}$ & \\
\hline $\begin{array}{l}\text { 10. Network simulators are very useful training tools } \\
\text { for academia. }\end{array}$ & \\
\hline $\begin{array}{l}\text { 11. The use of network simulators promotes the } \\
\text { motivation, creativity and self-learning capacity of }\end{array}$ & \\
\hline
\end{tabular}




\begin{tabular}{l|l|}
\hline students. & \\
12. Network simulators can be very useful tools for & \\
the professional field. & \\
\hline
\end{tabular}

The statistics with respect to the third item show a total agreement with regard to the content of the practice and their relationship with the theoretical concepts explained in lectures.

According to the opinions collected regarding the fourth premise, students were generally quite satisfied with the knowledge and skills acquired in the practice sessions, as only $15 \%$ of them partially disagreed.

The opinions of the students regarding the fifth statement corroborate authors' thought about the ease of use of the selected simulation tools, although most of the students used PT. Similarly, the pie chart for the sixth aspect reflects the overall satisfaction regarding the assimilation of the theoretical contents by using network simulation.

According to the results obtained from the seventh item, students were, in general, satisfied with the degree of autonomy and flexibility provided by the experimentation through simulation. Specifically, almost half of them completely agree with this statement. "Thanks to this methodology, I have been able to do and complete the practicals", stated a student who combined work with studies and could not physically attend lectures and laboratory sessions. We think that $8 \%$ of the students that partially disagreed with this aspect were students having full flexibility for completing their practicals (either physically in the laboratory with real equipment or through simulation). The results collected regarding the similarity with working with real equipment (aspect 8) are more disparate. $10 \%$ of the students preferred not to evaluate this aspect. This might occur mainly because some students had little or no experience working with real equipment. In addition, some of the students had not attended any practical in the laboratory because of attendance dispensation due to incompatible timetables/scheduling with their work. Negative scores were quite small: $1 \%$ of the students totally disagreed and $12 \%$ partially disagreed. A probable cause of the users' dissatisfaction with regard to this aspect might be that most of them used PT, with which they could only experiment with Cisco equipment. It is also important to note that although the configuration tasks are fairly reliable, other tasks such as the physical mounting, wiring and equipment handling are extremely simplified by using network simulation. However, a high percentage of the students showed to be satisfied.

The results gathered from the ninth item show an almost complete satisfaction (92\%) on the experience gained through the use of network simulation, with $41 \%$ of students being fully satisfied.

We can observe that $58 \%$ of the students totally agreed with the convenience of the network simulators as a learning resource (tenth item), $35 \%$ partially agreed, only $7 \%$ partially disagreed, and no students totally disagreed.

The results obtained from aspect 11 are also very gratifying, since $83 \%$ of the students agreed with the advantages provided by network simulators to stimulate motivation, creativity and self-learning skills. A marginal percentage of 2 $\%$ disagreed with these statements.

Finally, the goal of the last aspect of the survey was to collect the students' opinion regarding the suitability of the simulators in the professional field. Although TAN is a subject of the second year of the Bachelor's Degree, as said before, it is also part of the adaptation course for graduate students from the previous curricula. Therefore, some of them have proved working experience as engineers in some companies. We think that $14 \%$ of students that preferred not to value this aspect mainly corresponded with those students without working experience in this field. However, more than $80 \%$ agreed with this item.

In general, it can be stated that students' impressions about the use of network simulators as a learning resource were very positive, since the survey results are a significant indicator of their satisfaction about the deployment of this methodology in the TAN subject. Note that the evaluation process is essential. It is not only valid to refine the employed methodology, but also it is fundamental to identify aspects to be improved in future courses, as well as to prioritize/plan future works using network simulation techniques.

Likewise, to corroborate the succes of this learning methodology, the results/scores obtained by the students' of the TAN subject should be compared with the ones of previous courses. However, this is not possible at this moment because this subject was coursed for the first time that year. Therefore, we cannot make use of direct precedents.


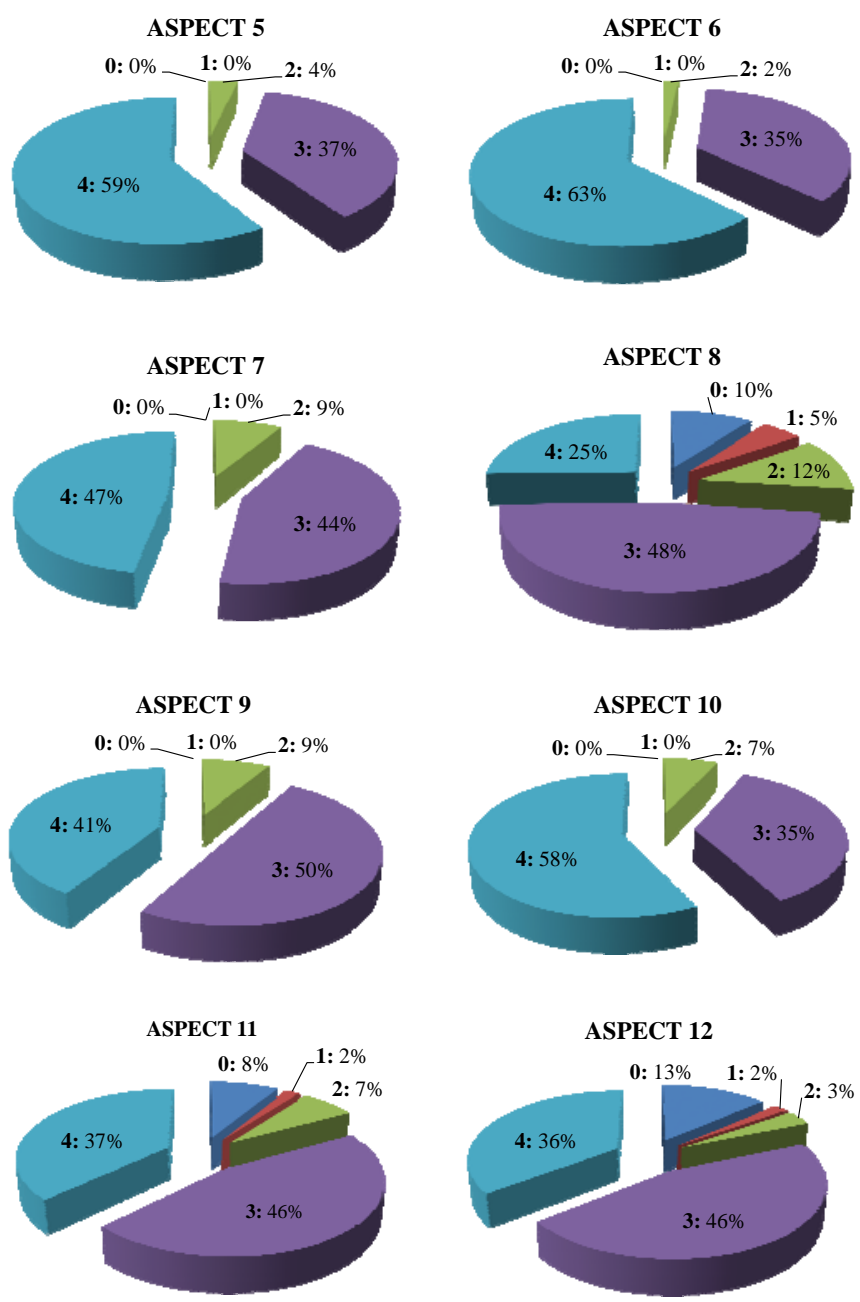

Fig. 1. Surveys Results

A possible consequence of the deployment of this methodology can be reflected in a higher percentage of students who take the final exam, as well as in the obtained scores, compared to the associated subject in the previous curricula, called “Telematics Networks and Services (TNS)". In particular, during the 2010/2011 academic year, $35 \%$ of the students enrolled in TSN did not attend the final exam, while $21 \%$ did not pass the exam. During the 2011/2012 academic year, the percentage of students that did not attend the final exam was reduced to $19 \%$, while the percentage of students that failed the exam was $14 \%$. This could be due to the higher degree of knowledge and skills acquisition, higher motivation and implication, as well as higher degrees of flexibility and autonomy provided by this innovative learning methodology.

It would not be appropriate to compare the marks given to practicals of both subjects (TAN and TSN) because both the program and the methodology have changed.

In the coming years, we will continue with the deployment of innovative learning methodologies in order to contribute to the improvement of the acquired skills and results by the students.

\section{CONCLUSIONS}

Within the EEHA, innovative learning resources must be adopted to motivate, in an active and integrated way, the implication, motivation, responsibility, creativity, autonomy and cooperation capacity of the students in their knowledge and skills acquisition processes. In this paper, we have reflected the importance of network simulators as learning tools to help the students to assimilate the theoretical concepts in a more flexible, relaxed and gradual manner, as well as to recognize their application to the professional field.

Consequently, a practice program has been elaborated for the TAN subject with the aim to allow the students to assimilate and experiment with the services offered by the network layer of the OSI model. Ther realization of the practice can be done through the use of both real equipment and simulation tools.

The use of network simulators as a learning resource has resulted to be very motivating, both for the students and instructors, allowing to complement and to consolidate the knowledge acquired in lectures and to recreate real use cases with which the students can experiment in an accurate and reliable manner.

Although the evidence base is not large (both the universe of subjects and the evaluation time are not optimal), the gathered impressions through surveys seem to give support to the use of network simulation in this area, as, in general, students have very positively scored the deployment of this training and learning methodology.

It is also important to note that the training of the students in network simulation will enable them to do their DFPs, as well as future Master Theses or research studies in the area of TE (e.g., multimedia communications, QoS, routing protocols comparison, etc.) using this kind of techniques, as it has been recently done in our university and research group.

Finally, even considering the multiple advantages provided by network simulation, it is important to emphasize that the use of this methodology cannot completely replace the laboratory tasks with real equipment (with which students will actually work during their professional careers), but simulation techniques must be considered as complementary support, training and coaching resources.

As future work, we will try to design and deploy other practice programs based on network simulation for other subjects of the third and fourth year of the TE area of the newly coursed Bachelor's Degree in Telecommunications at our University. We also plan to use simulation techniques in future research studies.

Another interesting future work consists in rigorously analyzing the benefits provided by the use of simulation, emulation or virtualization techniques for students in various teaching and professional fields, not only in the TE area, from the point of view of both the acquisition of practical skills and intellectual training. 


\section{REFERENCES}

[1] J. Lloret, J.M. Jimenez, F. Boronat, J. Tomas y J.R. Diaz, “Utilización de diversas metodologías didácticas para desarrollar las habilidades de los estudiantes de Ingeniería Técnica de Telecomunicaciones”, Congreso Internacional de Docencia Universitaria e Innovación (CIDUI), Barcelona (Spain), July 2006.

[2] R. Lacuesta y C. Catalán, “Aprendizaje Basado en Problemas: Una experiencia interdisciplinar en Ingeniería Técnica en Informática de Gestión”, Actas de las X Jornadas de Enseñanza Universitaria de la Informática (JENUI 2004), Madrid (España), July 2004.

[3] S. Cabrero, X. G. Pañeda, R. García, D. Melendi, R. Orea, "Herramienta educacional para el diseño y configuración de redes de comunicaciones”, Revista Iberoamericana de Tecnologías del Aprendizaje (IEEE RITA), Vol. 5, Núm. 1, February 2010.

[4] M. Riesco y M. Díaz, "Sistema Docente de Realimentación Inmediata en Clases Prácticas”, XI Jornadas de Enseñanza Universitaria de la Informática (JENUI 2005), Alicante, España, July 2005.

[5] S. Sendra, A. Cánovas, M. García, J. Lloret, “Cooperative assessment in the hands on skills of computer networks subjects”, IEEE EDUCON Education Engineering 2010 - The Future of Global Learning Engineering Education, Madrid (España), April 2010.

[6] C. Goldstein, K. Stark, S. Leisten, and A. Tickle, "Effectively Using a Network Simulation Tool to Enhance Student's Understanding of Computer Networking Concepts”, Capítulo IV del libro “Tools for Teaching Computer Networking and Hardware Concepts”, Editor: Nurul Sarkar, Editorial: IGI Global, February 2006, 386 pages, ISBN13: 9781591407355, EISBN13: 9781591407379.

[7] http://www.opnet.com/, Last Access in July 2012.

[8] http://www.omnetpp.org/, Last Access in 2012.

[9] http://www.isi.edu/nsnam/ns/, Last Access in 2012.

[10] http://nsl.csie.nctu.edu.tw/nctuns.html, Last Access in 2012.

[11] http://www.cisco.com/web/learning/netacad/course_catalog/PacketTrace r.html, Last Access in 2012.

[12] http://www.gns3.net/, Last Access in 2012.

[13] M. Garcia, F. Boronat, y J. Lloret, "Network simulators comparative from the educational point of view”, International Technology, Education and Development Conf., Valencia (Spain), March 2008.

[14] A. García, L.M. Escobar, A. Navarro y A. Vásquez, "Método de evaluación y selección de herramientas de simulación de redes”, Revista Sistemas y Telemática, Vol. 9, No.16, 55-71, March 2011.

[15] N. I. Sarkar y S. A. Halim, "Review of Simulation of Telecommunication Networks: Simulators, Classification, Comparison, Methodologies, and Recommendations”, Journal of Selected Areas in Telecommunications (JSAT), pp. 10-17, March 2011.

[16] http://www.cisco.com/web/learning/netacad/index.html, Last Access in 2012.

Mario Montagud Mario Montagud was born in Montitxelvo (Spain). He studied Telecommunications Engineering at the Polytechnic University of Valencia (UPV). Since then, he is researching on Computer Networks, Multimedia Systems, Synchronization Protocols and Simulation Techniques. He has been an IEEE member since 2009. He is also member of the Technical Committee of several international conferences and of the Editorial Board of international journals.

Dr. Fernando Boronat was born in Gandia (Spain), and went to the Polytechnic University of Valencia (UPV) in Spain, where he studied Telecommunications Engineering. After working for Spanish Telecommunication Companies he moved back to the UPV in 1996, where he is an Assistant Professor in the Communications Department at the Gandia Campus. He obtained his phD degree in 2004 and his topics of interest are Communication networks, Multimedia systems, Multimedia Protocols and synchronization. He has been an IEEE member since 1993 and is involved in several IPCs of national and international journals and conferences.

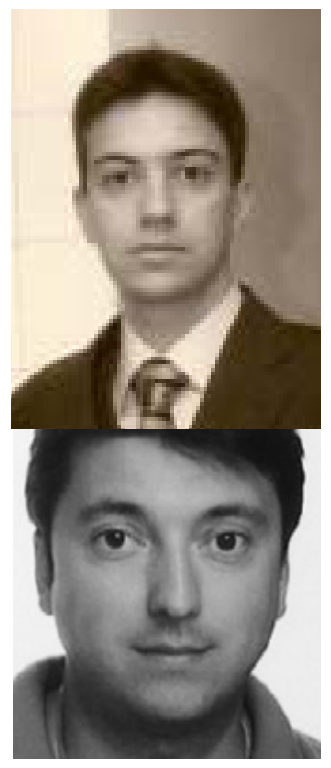

